\title{
Collaborative Aerial-Drawing System for Supporting Co-Creative Communication
}

\author{
Akihiro OSAKI $^{* *}$, Hiroyuki TANIGUCHI ${ }^{* * *}$ and Yoshiyuki MIWA ${ }^{* * * *}$ \\ ${ }^{* *}$ Wabot-House Laboratory, Waseda University, \\ 3-4-1 Ookubo, Shinjuku-ku, Tokyo, 169-8555, Japan \\ E-mail: osaki@aoni.waseda.jp \\ *** Graduate School of Science and Engineering, Waseda University, \\ 3-4-1 Ookubo, Shinjuku-ku, Tokyo, 169-8555, Japan \\ E-mail: h-taniguchi@akane.waseda.jp \\ ****Faculty of Science and Engineering, Waseda University, \\ 3-4-1 Ookubo, Shinjuku-ku, Tokyo, 169-8555, Japan \\ E-mail: miwa@waseda.jp
}

\begin{abstract}
This paper describes the collaborative augmented reality (AR) system with which multiple users can handwrite 3D lines in the air simultaneously and manipulate the lines directly in the real world. In addition, we propose a new technique for co-creative communication utilizing the $3 \mathrm{D}$ drawing activity. Up to now, the various 3D user interfaces have been proposed. Although most of them aim to solve the specific problems in the virtual environments, the possibility of the 3D drawing expression has not been explored yet. Accordingly, we paid special attention to the interaction with the real objects in daily life, and considered to manipulate real objects and 3D lines without any distinctions by the same action. The developed AR system consists of a stereoscopic head-mounted display, a drawing tool, 6DOF sensors measuring three-dimensional position and Euler angles, and the 3D user interface, which enables to push, grasp and pitch 3D lines directly by use of the drawing tool. Additionally users can pick up desired color from either a landscape or a virtual line through the direct interaction with this tool. For sharing 3D lines among multiple users at the same place, the distributed-type AR system has been developed that mutually sends and receives drawn data between systems. With the developed system, users can proceed to design jointly in the real space through arranging each 3D drawing by direct manipulation. Moreover, a new application to the entertainment has become possible to play sports like catch, fencing match, or the like.
\end{abstract}

Key words: 3DUI, Drawing System, Augmented Reality, Communication

\section{Introduction}

Drawing activities possess an important role as the bodily expression that can intuitively express not only the transmission of symbol information such as the characters and shapes, but also the dimension and depth of the spatial space. In addition, the drawing activities can be used by wide range of user's age, so that it is effective and versatile expression means for communication in daily life. On the other hand, three-dimensional (3D) drawing technique has been mainly used for designing machines and architectures known as 3D CAD, which, in these days, is widely used in the entertainment industry including animation and film making using 3D characters and/or 3D images.

If the $3 \mathrm{D}$ drawing space is expanded from the restricted desktop pc to the real world, 
one can draw pictures using his whole bodily actions and many people can simultaneously perform drawing in the air during their routine activities. It is, then, expected that $3 \mathrm{D}$ drawing can offer a novel co-creative communication system which utilizes spatial space around them as an effective communication media.

In order to display some painting objects in the air, the augmented reality(AR) technique seems to be an effective technique, which overlay the virtual objects onto the real world by using a video or optical see-through device ${ }^{(1)}$. And, until now, some applications have been suggested that make multiple people intuitively share the complicated information in the real world, such as a map ${ }^{(2)}$ or a scientific data ${ }^{(3)}$. In addition, it is not only a presentation technique of such information, and use for entertainment is also proposed, which multiple people enjoy to interact with a virtual character and the object together ${ }^{(4)}$. And it has been shown the possibility that the width of communications in a real space is expanded. Moreover, a lot of use as the three-dimensional modeling tool is done, and it has been suggested the possibility that the width of the human expression including the design is expanded more ${ }^{(5)(6)}$.

However, many of these tools were that the user was made to design by combining primitive shape including the hexahedron and the sphere provided beforehand like the block. On the other hand, in this study, we assume to make the user to paint in the air based on the AR technique as if the line came out from the hand without primitive shapes. Therefore, it has possibilities to expand the range of expression for supporting co-creative communication so that the user can make something from primitive object by drawing improvisationally on site. Furthermore, it is expected that the multiple people can communicate with sharing a spatial sense in each other via getting into the drawn picture existing in the real world as if they may meet in their-self cave painting.

How can we draw 3D pictures in a real space? The drawing object depends on a viewing position, which is characteristic problem in an immersive-type $3 \mathrm{D}$ drawing. Can we, then, establish an effective communication through drawing possessing these problems? More specifically, a drawing person paints pictures including the depth direction which, in turn, becomes a dead angle for him. It is, therefore, reported that there are propensities that the line position in depth direction will become unstable and drawn lines constituting a picture will be separated while going further into the depth direction ${ }^{(7)}$.

Therefore, in collaborative $3 \mathrm{D}$ drawing, if the above failure left unsolved, the collaborator looking from different viewpoint is hard to understand what is drawn. It follows that it can cause the misunderstanding in each other. Therefore, under this situation and particularly during the collaborative drawing, a drawing person A is observing a picture having been drawn by a person B from A's viewing point which is different from the B's. As a result, a message on what a person $\mathrm{A}$ intends to draw is not precisely transmitted to a person $\mathrm{B}$, and the collaborative drawing could be continued with carrying these unsolved problems.

For this reason, it becomes a crucial issue to construct an effective interface functioning between users, which can correct failed lines properly and delete them if necessary.

Up to now, numerous 3D user interfaces have been suggested to support 3D drawing. Most of them, however, concern only virtual environment, and there is very a few interface applicable to the real world.

On the aforementioned backgrounds, we specified our aim to establish the co-creative human communication system through the collaborative 3D drawing in the real world. For achieving the aim, we paid, at first, our attention to the Mixed reality (MR) technique as 3D drawing technique applicable to the real space. Then, we developed and constructed a distributed-type 3D drawing system, with which a plurality of people can draw and overlap virtual lines simultaneously into a real space to participate to complete a picture. 
Assuming that participants who draw lines and pictures can continue their task while they are correcting wrong lines in site and checking their corrections, we developed an interface, with which they can manipulate virtual lines on a real world directly by their hands.

In the following sections, the details on the developed system will be described and several demonstrations will be introduced to show how the system was applied to the collaborative drawing performance.

\section{3D User Interfaces}

In the past, numerous techniques for manipulating objects in virtual space have been proposed $^{(8)(9)}$. Especially in 3D drawing, which possesses a difficulty to position the 3D lines properly at a certain position, several interface systems aiming on the bodily actions have been suggested. For example, there is a superimposing technique of the virtual image over the real objects, so that the virtual object can follow the manipulation on the real substance ${ }^{(10)}$. With this superimposing technique, although movements of real substance can be corresponded to those of the virtual substance, the technique does not take their relationships in shapes into consideration. However, it was noticed that routine activities such as hand-manipulation can be directly applied to the technique, and effectiveness and efficiency of such hand-manipulation have been recognized.

In addition, many other techniques using bodily actions in daily life are suggested and its effectiveness is confirmed. For example, there is a technique for manipulating the virtual objects directly by a tangible tool such as a pair of kitchen tongs ${ }^{(11)}$, and a bimanual technique which treats a virtual 3D line as a sort of tape ${ }^{(12)}$. However, these techniques intend to manipulate an objectified space where drawing person (residing in a real world such as workbench or the desktop monitors) cannot enter. Hence, they do not consider any manipulations to space which is non-separable from the drawing person.

More specifically, when drawing picture is exercised by combining the real space and virtual space, a smooth communication and transmitting information between these two spaces are expected during the drawing activity. However, the aforementioned interface techniques might cause un-harmony and separation feelings between two spaces, resulting in that the real space and virtual space are separated and only virtual drawing can be achieved.

For preventing these problems, the authors paid attention to progressive activities during a picture drawing. Namely, a drawing person integrates visually his surrounding scenes and virtual lines to gradually complete his art work, regardless of real expression or virtual expression of his objects. In other words, the drawing person selects appropriate painting material and corrects his expression, all the time during his art work, through bodily interactions with his surrounding atmosphere comprising real and virtual spaces. Therefore, it is necessary for him to match his bodily interaction to the real space with that to the virtual space.

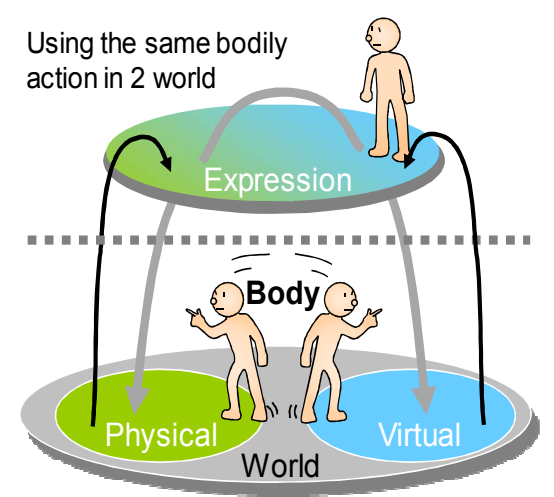

Fig. 1 Concept of the user interface for 3D drawing in the real world 
Based on the above concept, as shown in Fig.1, we developed an interface. Using the interface, one can pick up painting materials, which can be used to either real or virtual space, through the same bodily movements. He can also correct unwanted drawn lines in a similar manner as he adjusts his art work by his hands in a real space. Actually we have mounted the interface portion (which can manipulate drawn lines by hand movements) to the previously developed immersive-type $3 \mathrm{D}$ drawing system ${ }^{(7)}$. The details are as follows.

\section{A. 3D drawing system}

For drawing 3D objects directly in the real world, we developed an immersive-type 3D drawing system comprising an stereoscopic optical see-through head-mounted display (HMD) which makes 3D lines visible as a virtual image, a general-purpose PC, and an interface which enables to pick up painting materials directly from the real object in the real world $^{(7)}$.

In this system, a user equipping with HMD on his head can handwrite 3D lines directly in the real world by moving a drawing tool in his hand (see Fig.2). The virtual 3D line is created with the OpenGL on the PC, based on (1) two three-dimensional position and Euler angle information obtained from magnetic sensors (Fastrak, Polhemus, Inc.) which are amounted on the drawing tool and the HMD, and (2) ON/OFF switch information of the drawing tools.
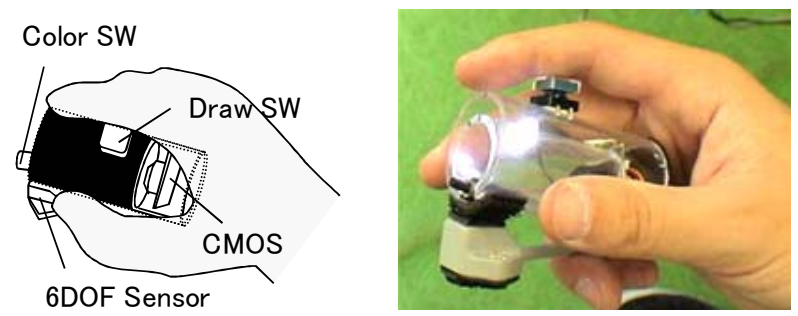

Fig. 2 Appearance of a Drawing Tool

Table 1 Specification of the hardware for the AR system

\begin{tabular}{r|r}
\hline Type & Stereoscopic Optical see-through HMD \\
\hline Weight & 262g (Headgear) \\
\hline Field of View & 30deg \\
\hline Display resolution & VGA (480*640) \\
\hline Video input & Wireless composite video (Microwave) \\
\hline 6 fof Sensor & FASTRAK, Polhemus, Inc. \\
\hline Drawing range & Radius of $760 \mathrm{~mm}(3050 \mathrm{~mm}$ max)Transmitter is center \\
\hline
\end{tabular}

The specifications of the HMD and sensor are listed in Table1.

For synchronizing a hand manipulation of lines and actual drawing during the drawing activity, the following flow (Fig.3) is followed to achieve the draw processing.

First, data on head and hand positions obtained by the magnetic sensors, line colors, thickness of lines, and conditions of two switches are packet-treated as the flag. Then, all of these data composing a packet is arranged chronologically per sampling, and saved to database (DB).

In the next step, a 3D collision incidence between the drawn line and a hand is judged per sampling, followed by that the movement processing for the contacting lined is done to the position data which is saved in the DB. Then, DB is accordingly uploaded.

At the final stage, 3D original lines are generated based on the thus updated DB. These lines are further transformed to the viewing coordination system which is corresponding to each both eyes of the drawing person for making the view in the three-dimensional 
appearance, and finally the line-images generated for each both eyes are transmitted to stereoscopic HMD wirelessly. As a result, the drawing person became possible to see the lines in binocular vision through the HMD.

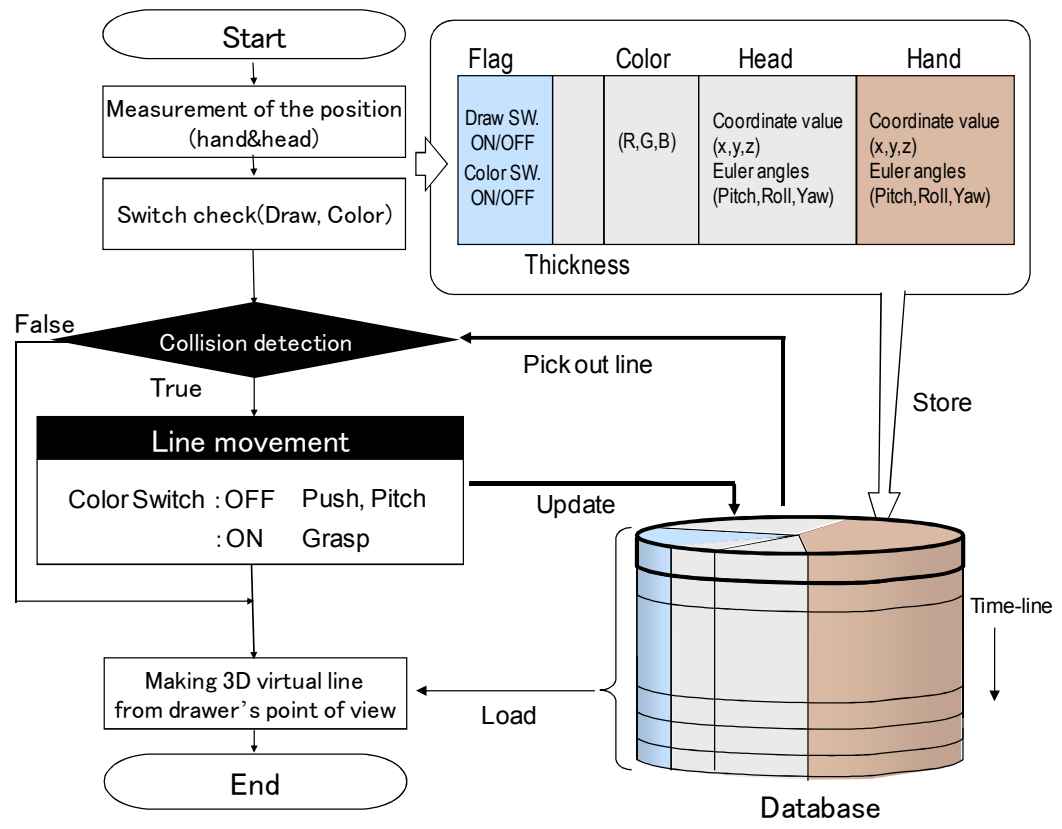

Fig. 3 Flow chart of the drawing included the manipulation

\section{B. 3D Collision Detection}

Manipulating processing for a 3D line contains the two steps: 3D collision detection between virtual 3D lines and drawing tool, and movement process of the 3D line corresponding to bodily movements.

For 3D collision detection as shown in Fig.3, the following criteria were set to position information on group of points comprising drawn lines saved in DB; (1) the position information is the nearest one to the drawing tool and (2) it is within satisfies these criteria is defined as the "collision point" and the drawn line convoluting these points is "collision drawn lines".

In addition, for a judgment for collision incidence, the judging range is displayed with 3D-wireframe box as a visual supportive expression(Fig.4). By changing the color of the frame box upon the collision incidence with the drawn line, which one of tools is in contact can be identified.
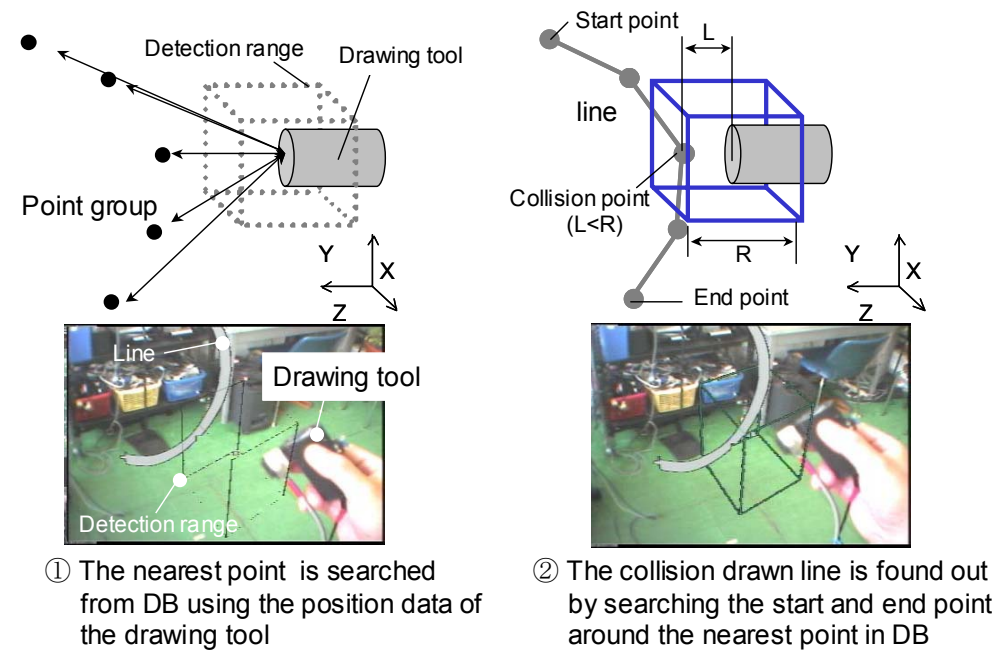

Fig. 4 Collision detection between the drawing tool and a virtual line 


\section{Manipulation Techniques}

We paid attention to hand movements when the real object is manipulated, and designed the user interface to have functions of 'push', 'grasp' and 'pitch' actions against a virtual line with bodily actions. These actions are done with drawing tools. A user holding the drawing tool can 'grasp' a 3D line by pushing the switch at its tip as if he manipulates the real objects with his thumbs and forefingers. Remaining two functions of 'push' and 'pitch' a 3D line can be done only by contacting the tool. The user can operate the real object and virtual objects with the same action (Fig.5). However, the collision detection doesn't function while the drawing is in progress. The details of each action are described below.

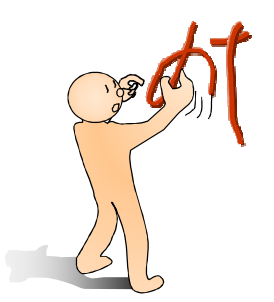

Grasping and moving a line to adjust the position directly

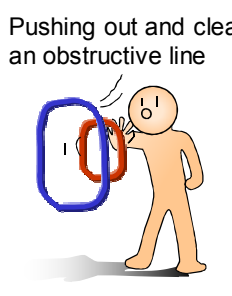

Fig. 5 Concept images about the usage of the interface for 3D drawing

1) Push

If the difference between the distance from the hand position at the collision moment to the collision point, and the distance from the hand position after passing through the line to the collision point are larger than the pre-determined threshold value, the distance passing through is added to all points of the "collision drawn line". This enables to move the lines with respect to the hand contact from any direction (Fig.6).
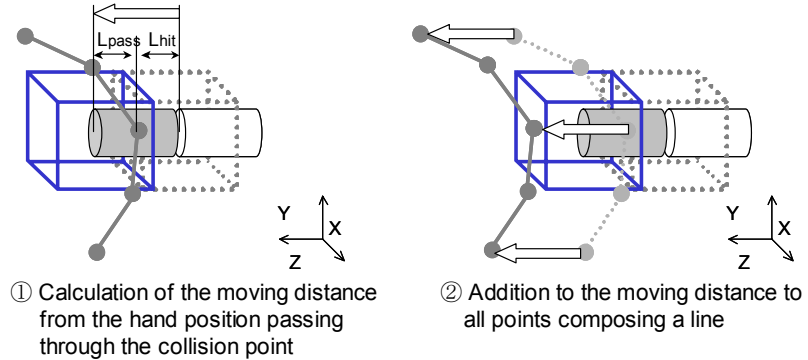

Fig. 6 Movement of a virtual line by "Push"

\section{2) Grasp}

In contrast with the 'push' processing of adding the distance of the hand passing through, 'grasp' movement can be achieved by matching the "collision drawn lines" with the position and angle of the hand.

While the color switch for obtaining the drawing tool is ON position at the contacting time of line, all points involved in the "collision drawn lines" are transformed into the local coordinate system which has a hand position as its center (Fig.7). By this process, the drawn line can be followed in response to translation and rotation of the hand movements.
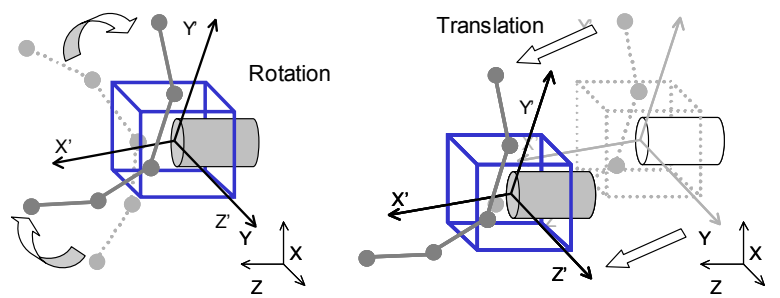

(1) When the Color SW is ON at the contacting (2) By turning off the Color SW, time of line, A line is transformed into the local you can finish grasping the line coordinate system centered on a hand.

Fig. 7 Movement of a virtual line by "Grasp" 


\section{3) Pitch}

If the collision is made at higher than the threshold speed (which is determined by the passing speed), it is defined as 'pitch' movement. By the 'pitch' processing, movement amount which was calculated at the 'push' processing portion is temporarily stored for each 'contacted drawn line' and is continuously added to point group comprising the drawn lines per sampling.

4) Picking up color

In order to pick up the painting materials directly from everything around users without any distinctions between real objects and virtual lines, desired color can be selected by pushing the color-obtaining button located at the tip portion of the tool onto the real substance. Moreover, the same color can be also obtained by pushing the button to the already colored drawn line directly.

Figure 8 outlines the processing for picking up colors. When the color switch in pushed down and a collision detection signal displays false, it should be indicated that it touches the real substance. Then, the color information is created by photographing the object with CMOS which is installed inside the drawing tool, and by image processing the photos ${ }^{(8)}$. On contrary, when the collision detection signal shows true, because the drawn line is on contact, the color information which is saved at the collision point can be set as the color information for the current drawn point.

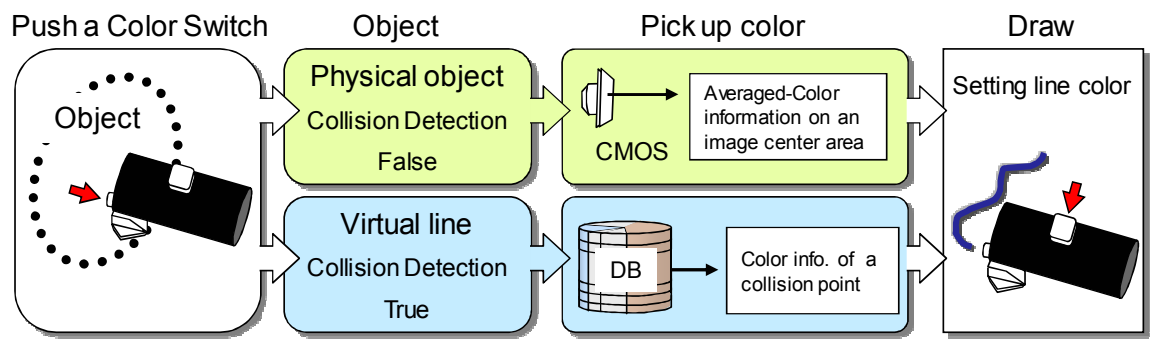

Fig. 8 Outline of picking up color with a real object or a virtual line

In the above, a user of this interface system can choose the color and thickness of lines and manipulate lines by his bodily actions, no matter what they are real or virtual lines (Table 2).

Table 2 Manipulation techniques for a physical object or a virtual line

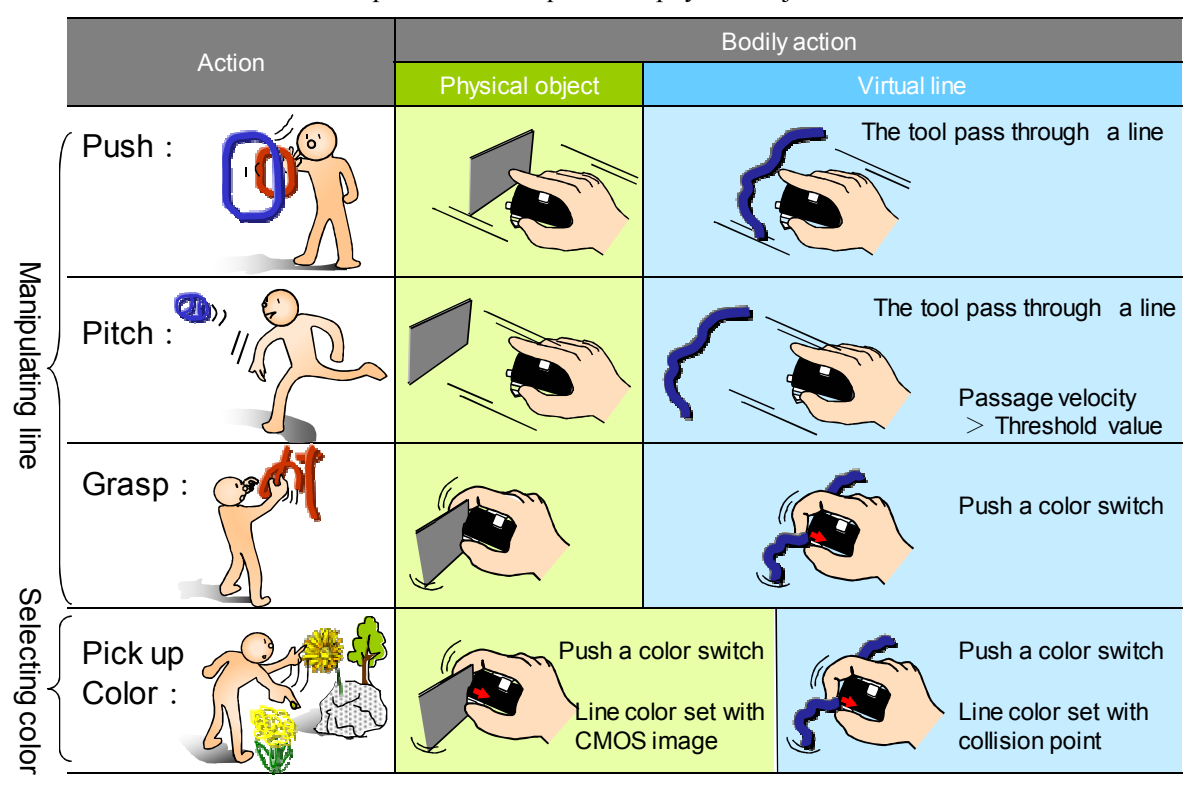




\section{Network System for Multi-User drawing}

For performing the 3D drawing in a collaborative manner, a distributed-type network system was constructed by LAN-connecting drawing systems (Fig.9). For collaborative drawing practice using the immersive-type 3D drawing system, position information on head and hand from both users are needed. Hence, magnetic sensors (3Space Inc., Fastrak) are employed which can perform simultaneous measurements on four data points on a same coordinate. The obtained information can be shared by P2P. The ON/OFF information on drawing tools of both users is also sent back and forth to generate respective DB on each drawing unit. They are also updated in real time base.

In order to manipulate a $3 \mathrm{D}$ line at the same time with the aforementioned network system, we have embedded a manipulation processing of 3D lines as well as building DB. However, because manipulation processing of drawn lines is performed individually for every sampling, a disagreement of timing during sending and receiving data between distributed-type systems may alter processing outcome.

Therefore, after data was updated, the exact same manipulation processing of 3D lines was performed for each system. As a result, the contents of the DB can be always identical among systems, and disagreement of drawing results can also be avoided.

Additionally if two users touch a same 3D line, a priority is given to the interface user who has a tool closer to the contact point. Finally, in each system, 3D lines are transformed to a user's view coordinate system which is based on a user's viewpoint corresponding to each system. As a result, two drawing persons can observe simultaneously a same line from different directions, and can manipulate simultaneously the line displayed on a real space. The sampling frequency of the above-mentioned processing was $15 \mathrm{~Hz}$. The time delay between each 3D drawing systems was less than one frame.

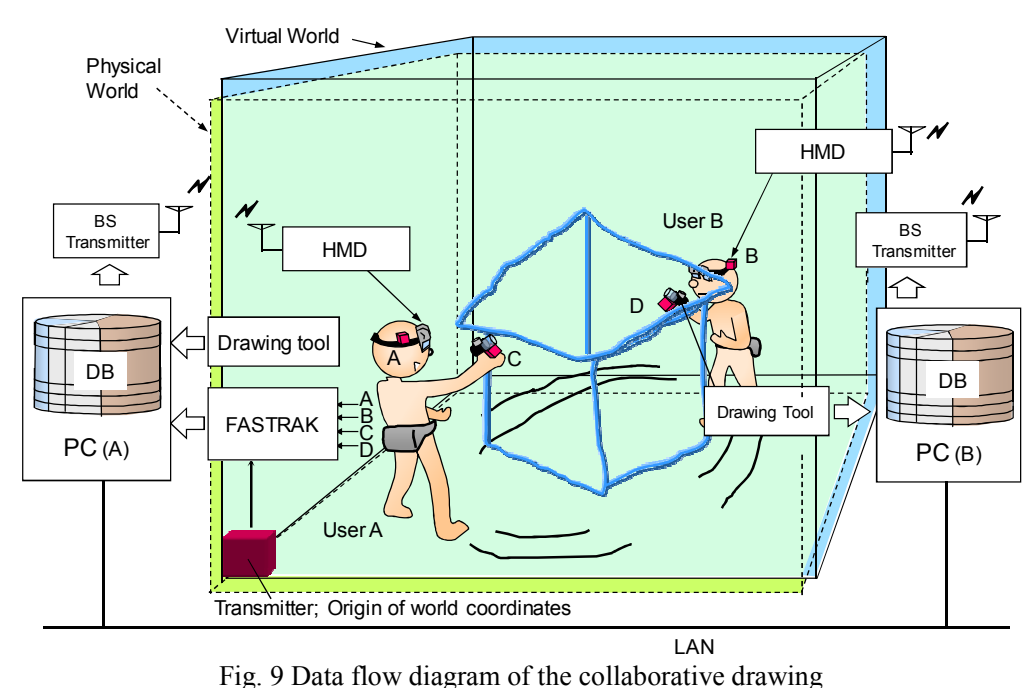

\section{Adjust and Erase 3D Line}

The developed interface makes it possible to correct failed portions of the 3D line by grasping and moving them, furthermore to erase unwanted portions by throwing them away. In this system, black 3D lines can be utilized as an eraser. This erasing function is realized because black 3D lines are seen as transparence from users with the distinctive effect of the half mirror on the optical-see-through type of HMD. However, this function works only when 3D lines to-be-erased are backside of black 3D lines. Several examples using the distributed-type system for 3D collaborative drawing are demonstrated in the following.

In immersive-type 3D drawing, as already mentioned, the line position is not stable in the depth direction of a user. Therefore, it is difficult to understand 3D images precisely 
from any other directions than the user's eyes. Even in such situation, as seen in Fig.10 for collaborative drawing, this interface systems allows the other user indicate the difference along the depth direction while observing the drawn lines from the side, so that the aforementioned failure can be prevented. Moreover, user A can correct failed lines which user $\mathrm{B}$ is drawing.

On the other hand, when a plurality of people is drawing things simultaneously at the same place, drawn lines are getting crowded and entangled, so that it becomes difficult to identify one line from the others. Therefore, after each user completed drawing lines at separate places, each user grasp them, move them, and put them together to finish their collaborative task. In addition, users can have an experience such as blocks assembling using drawn lines or components which were previously pushed away and kept at the corner of their surrounding spaces.
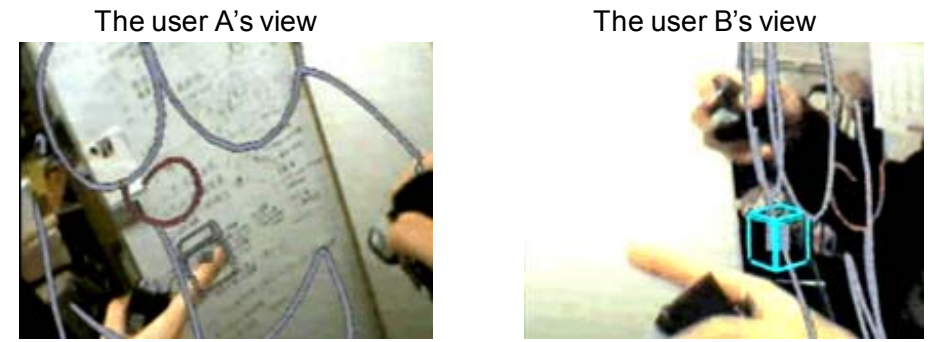

A instructs $B$ to the depth direction from the side position
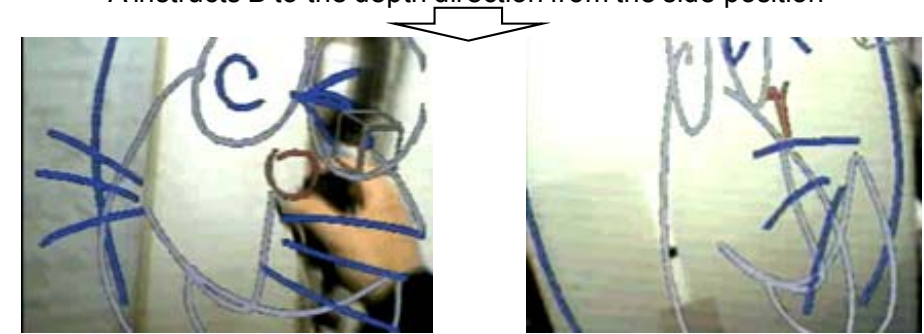

Several parts are painted an inappropriate position

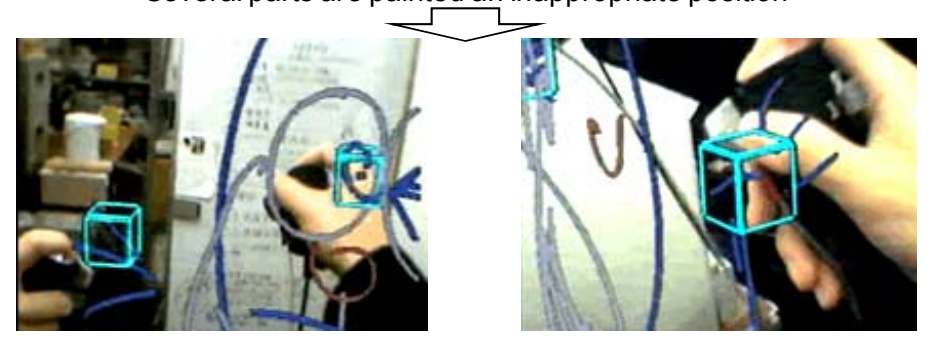

Two people adjust the position at the same time

Fig. 10 Screen shot of the adjustment position collaboratively

As an application of this system, Fig.11 demonstrates two persons participating flower arrangements. As seen in Fig.11, they are able to draw leaves and flowers individually and arrange these $3 \mathrm{D}$ images into a same vase. This demonstration suggests us that a new expression other than 3D drawing can be established.

Furthermore, because users are able to acquire desired colors from 3D drawn lines, they can arrange the color information, which is previously obtained from real substances, on a plurality of real spaces to make their own color pallet board. Even when a user A does not have desired color available for him, he can ask a user B to throw the color, so that they can share the color information.

From the above, interface users can not only express their idea by handwriting lines, but also obtain painting materials or manipulate lines freely through the bodily interaction with their surrounding spaces. 


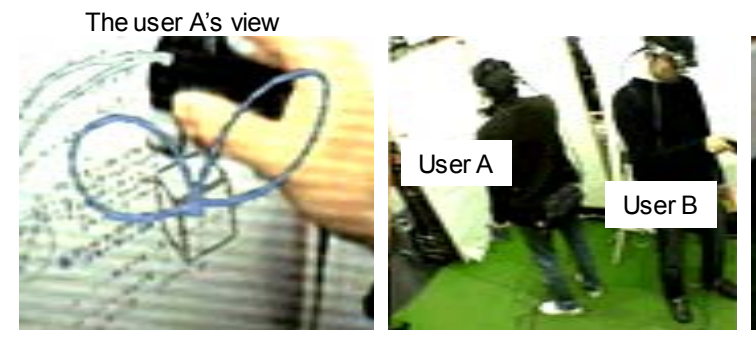

(1) Two users draws flowers individually

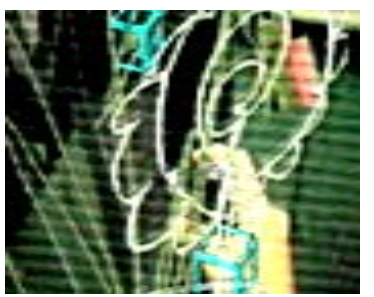

(2) Two users arranges flowers in the shared vase
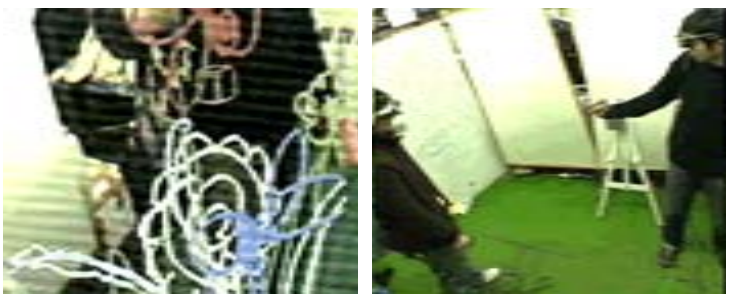

The user B's view

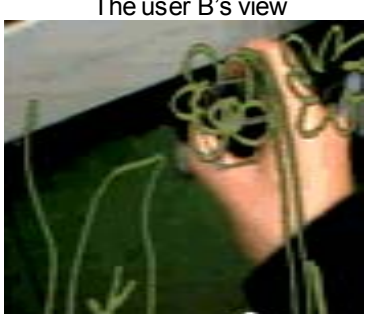

$\sqrt{2}$
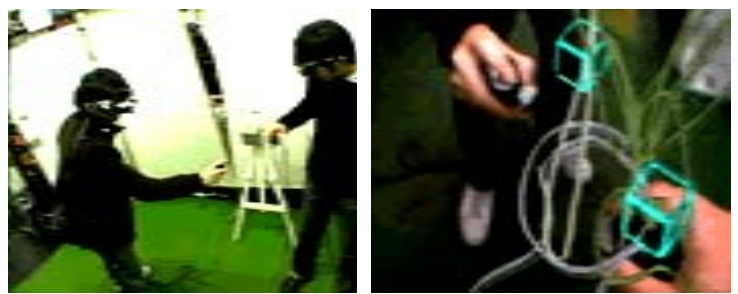

$\sqrt{2}$

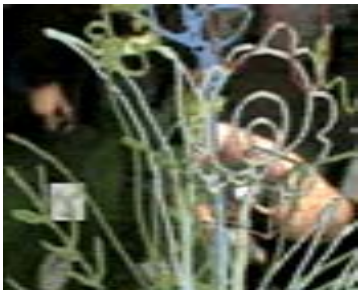

(3) Two users appreciate the flower arrangement from a different direction

Fig. 11 Screen shot of a combination with some drawings

\section{User Testing}

A collaborative demonstration was conducted by 6 pairs of 20-24 year male participants. Although each participant is individually familiar with this interface system, they have never participated to the collaborative drawing. The demonstration task required the participants drawing jointly to complete one scene in the air. There were two cases; with and without the manipulation interface. Then, we observed their behavior during the drawing, and interviewed to the participants about the difference of the drawing feeling with or without the interface after a completion of the drawing scene.

In case of without the interface, it is often observed that users concentrated on their own drawing and were not able to comprehend others' drawings. In consequence, it was hard to communicate what they drew each other, and there were many incidents observed that two users do not share a role for collaborative drawing one picture, but rather draw their own pictures independently.

On the other hand, in case with interface, at first, we noticed bodily interactions using lines rather than drawing 3D pictures jointly. In this demonstration, this tendency was observed by 4 pairs in 6 pairs. For example, it was often observed that each pair drew heart symbols, balls and some characters, and threw these messages to each other. In addition, other actions of grasping lines were observed, like drawing a bat and swinging it, or drawing a sword or a bar and swinging them(Fig.12). The interaction was done to not only own lines ( $6 / 8$ people) but also the other's lines ( $5 / 8$ people) . They seemed to anticipate a certain situation throughout the whole appearance of drawn products, and added some necessary liens and parts to make it better.

It was also found that, right after they finished each drawing line, their bodily movements are noticed and these movements tended to more active by continuing their art work further. These activities were not observed for the case of without the interface. When 
further detailed analyses on their activities were done, the following results were obtained.

They started with throwing or swinging a sort of abstract substance, and gradually their imagination and progressive creation changed to a concrete picture, as seen in Fig.12. Furthermore, a user can catch the ball thrown by the other user nicely by judging the direction and speed of the coming ball. It was also observed that two users handled the tools nicely to contact their swords even though they did not feel any repulsive force at the time when their swords were in contact with each other. Namely, users intensified their co-sharing of movement images throughout drawing a picture. "I actually sense a feeling of throwing a ball." "I feel as if I swing a bar." These are just few among many positive comments, supporting the above analysis.

Introducing the drawing manipulation, as has been described in the above, is effective to correct and/or delete drawn substances during the collaborative designing. It was also suggested that it is an effective and supportive means to create a novel communication. In other words, through the manipulating drawn lines or pictures, necessary tools can be generated in site, and users can use and play with the generated tools. They can also throw back and forth messages which were drawn in the air. All these communication activities can be realized in an improvisation manner.

Furthermore, we received an interesting comment; "I wonder if I can throw the ball against the wall?" This is again just one example among many similar comments related to contacting of real world and drawn lines, suggesting a possibility for further activities in not only the virtual space but also the surrounding atmosphere.

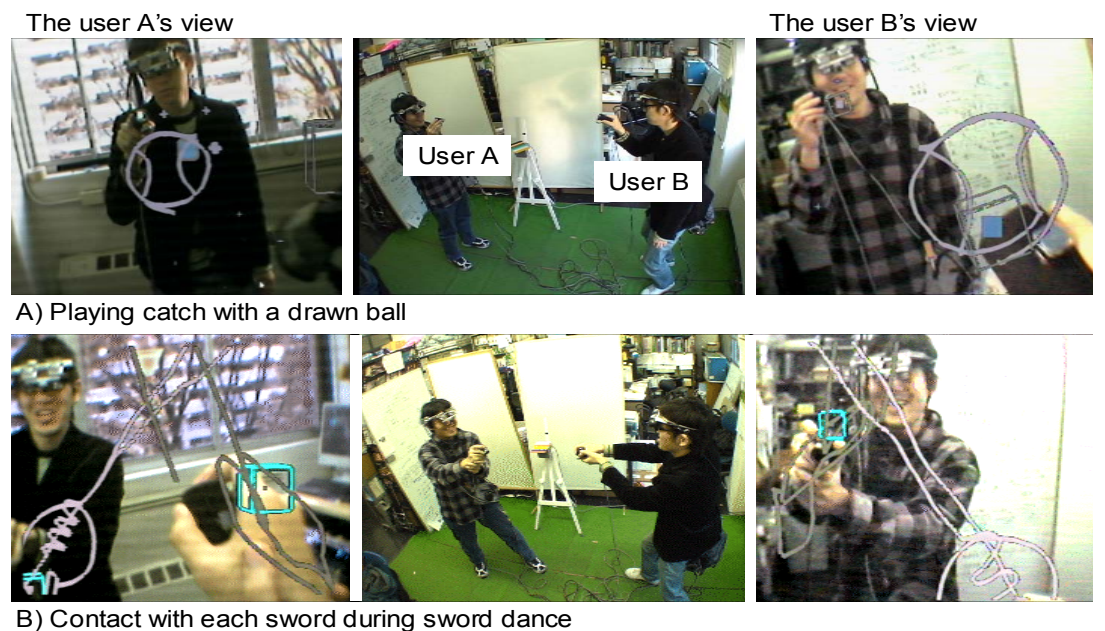

Fig. 12 Screen shot of the playing by using a picture as a tool

\section{Conclusion}

In this study, we developed the distributed-type 3D drawing system with embedded direct manipulation interface so that the co-creative human communication can be performed through the $3 \mathrm{D}$ drawing activities in the real world. Such interface performs similar actions to obtain painting materials or adjust drawn lines, in both real and virtual spaces, so that 'push', 'grasp', or 'pitch' actions on virtual lines can be achieved by hands. Moreover, by the interface system, not only color information can be obtained from the real substance, but also the color information can be acquired from the virtual lines in a similar manner as for the real lines.

With the developed system, two people correct and/or delete virtual lines collaboratively in site. Bodily interactions with the drawn lines by throwing them by hands, or swinging them can be realized (see Fig.13). Hence, participants can enjoy improvisation plays in the real space. Since participants can perform not only their collaborative art woks, but also a series of activities including communication by using hands, the creation activity 
can be widened. Lastly, it is expected that the communicability can be enhanced through a variety of bodily interactions. On the other hand, because the above-mentioned result was confirmed in the limited environment and the situation, it is necessary for investigating the effect for co-creative communications through this drawing in the air on various situations and sites in the future. Therefore the authors plan to evaluate the creation process of this communications quantitatively in addition to constructing an applied system corresponding to each situation based on this drawing interface.
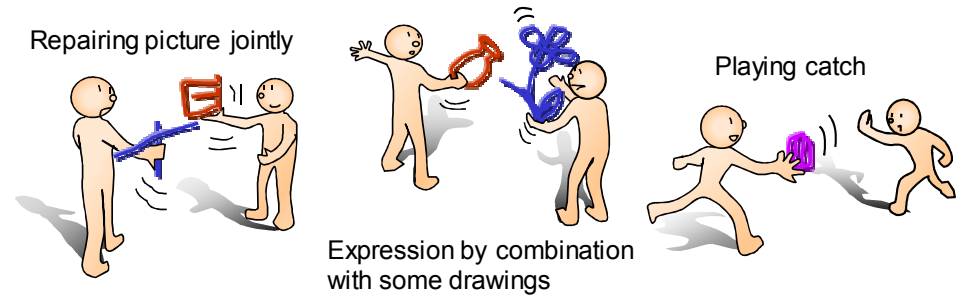

Fig. 13 Communications achieved through the developed system

\section{Acknowledge}

This study was funded in part by WABOT-HOUSE Project, and has been conducted under the project "Generation and Control Technology of Human-Entrained Embodied Media" and is supported by CREST of JST.

\section{References}

(1) Feiner, S.K., “Augmented reality: a new way of seeing,” Scientific American, Vol. 4 No.24, 2002, pp.48-55.

(2) Hedley, N.R., Billinghurst, M., Postner, L., May, R. and Kato, H., "Explorations in the use of augmented reality for geographic visualization," Presence, Vol. 11 No.2, 2002, pp.119-33.

(3) Fuhrmann, A., Löffelmann, H., Schmalstieg, D. and Gervautz, M., "Collaborative Visualization in Augmented Reality," IEEE Computer Graphics and Applications, 18(4), 1998, pp.54-59.

(4) Ohshima, T., Satoh, K., Yamamoto, H. and Tamura, H., "AR2 Hockey: A Case Study of Collaborative Augmented Reality,” Proceedings of IEEE VRAIS '98, 1998, pp.268-275.

(5) Butterworth, J., Davidson, A., Hench, S. and Olano, T. M. "3DM: a three dimensional modeler using a head-mounted display," Proceedings of the 1992 symposium on Interactive 3D graphics, 1992, pp.135-138.

(6) Kiyokawa, K., Takemura, H., Katayama, Y., Iwasa, H. and Yokoya, N., "VLEGO: A Simple Two-handed Modeling Environment Based on Toy Blocks," Proceedings of ACM Symposium on Virtual Reality Software and Technology 1996 (VRST'96), 1996, pp. 23-34.

(7) Osaki, A., Ebisawa, H. and Miwa, Y., "Drawing Interface for Creating Embodied Space in the Real World," Proceedings of RO-MAN2004, 2004, pp. 199-204.

(8) Hand, C., "A Survey of 3D Interaction Techniques," Computer Graphics Forum, 16(5), 1997, pp. 269-281.

(9) Bowman, D. et al., "3D user interfaces: theory and practice," Addison-Wesley, 2004.

(10) Broll, W., Meier, E., Schardt, T., "The Virtual Round Table a Collaborative Augmented Multi-User Environment," Proceedings of the ACM CVE2000, 2000, pp. 39-46.

(11) Schkolne, S., Pruett, M. and Schröder, P., "Surface Drawing: Creating Organic 3D Shapes with the Hand and Tangible Tools," Proceedings of the SIGCHI2001, 2001, pp. 261-268.

(12) Grossman, T., Balakrishnan, R., Kurtenbach, G., Fitzmaurice, G., Khan, A. and Buxton, B., "Creating Principal 3D Curves with Digital Tape Drawing," Proceedings of the SIGCHI2002, 2002, pp. 121-128. 